\title{
THREE TIME SCALE SINGULAR PERTURBATION PROBLEMS AND NONSMOOTH DYNAMICAL SYSTEMS
}

\author{
BY
}

PEDRO T. CARDIN (Departamento de Matemática - Faculdade de Engenharia de Ilha Solteira, UNESP - Univ Estadual Paulista, Rua Rio de Janeiro, 266, CEP 15385-000 Ilha Solteira, São Paulo, Brazil),

PAULO R. DA SILVA (Departamento de Matemática - Instituto de Biociências, Letras e Ciências Exatas, UNESP - Univ Estadual Paulista, Rua Cristóvão Colombo, 2265, CEP 15054-000 S. J. Rio Preto, São Paulo, Brazil),

MARCO A. TEIXEIRA (IMECC-UNICAMP, CEP 13081-970, Campinas, São Paulo, Brazil)

Abstract. In this paper we study three time scale singular perturbation problems

$$
\varepsilon x^{\prime}=f(\mathbf{x}, \varepsilon, \delta), \quad y^{\prime}=g(\mathbf{x}, \varepsilon, \delta), \quad z^{\prime}=\delta h(\mathbf{x}, \varepsilon, \delta),
$$

where $\mathbf{x}=(x, y, z) \in \mathbb{R}^{n} \times \mathbb{R}^{m} \times \mathbb{R}^{p}, \varepsilon$ and $\delta$ are two independent small parameters $(0<\varepsilon, \delta \ll 1)$, and $f, g, h$ are $C^{r}$ functions, where $r$ is big enough for our purposes. We establish conditions for the existence of compact invariant sets (singular points, periodic and homoclinic orbits) when $\varepsilon, \delta>0$. Our main strategy is to consider three time scales which generate three different limit problems. In addition, we prove that double regularization of nonsmooth dynamical systems with self-intersecting switching variety provides a class of three time scale singular perturbation problems.

1. Introduction. The present work fits within the geometric study of singular perturbation problems expressed by two parameters families of vector fields on $\mathbb{R}^{\ell}$. A classic singular perturbation problem is expressed by a differential equation $z^{\prime}=h(z, \varepsilon)$ with $z \in \mathbb{R}^{n+m}, \varepsilon \geq 0$ and $h \in C^{\infty}$, where we want to study the phase portrait for small values of $\varepsilon>0$ near a set that contains a manifold of singular points of $z^{\prime}=h(z, 0)$. Let $z=(x, y) \in \mathbb{R}^{n+m}$ and $f, g$ be smooth functions. We deal with equations that may be

Received October 12, 2012.

2010 Mathematics Subject Classification. Primary 34D15, 34N05, 34C20.

Key words and phrases. Geometric theory, singular perturbations, three time scales, nonsmooth dynamical systems.

E-mail address: pedrocardin@mat.feis.unesp.br

E-mail address: prs@ibilce.unesp.br

E-mail address: teixeira@ime.unicamp.br 
written in the form

$$
x^{\prime}=f(x, y, \varepsilon), \quad y^{\prime}=\varepsilon g(x, y, \varepsilon) .
$$

The main trick in geometric singular perturbation theory consists in considering the family (11) in addition with the family (2) obtained after the time rescaling $t=\varepsilon \tau$

$$
\varepsilon x^{\prime}=f(x, y, \varepsilon), \quad y^{\prime}=g(x, y, \varepsilon) .
$$

Equation (11) is called the fast system and (2) is the slow system. Observe that for $\varepsilon>0$ the phase portraits of the fast and slow systems coincide. For $\varepsilon=0$ let $\mathcal{S}$ be the set of all singular points of (11). We call $\mathcal{S}$ the slow manifold of the singular perturbation problem, and it is important to notice that equation (2) (with $\varepsilon=0$ ) defines a dynamical system on $\mathcal{S}$, called the reduced problem. Combining results on the dynamics of these two limiting problems, one obtains information on the dynamics for small values of $\varepsilon$.

When systems present a clear separation in time scales, methods of approximations of slow-fast systems can be applied. Around 1980, geometric singular perturbation theory was introduced. The foundation of this theory was laid by Fenichel [3] (see also [19]) and essentially uses geometric methods from dynamical systems theory for studying the properties of solutions of the system.

Singularly perturbed systems arise in a great variety of areas, as for instance traveling wave problems in reaction-diffusion equations, enzyme kinetics, the propagation of action potentials in neurophysiology, perturbed Hamiltonian systems, control theory, coupled mechanical oscillators, etc. For a more complete introduction about the techniques which led to a geometric analysis of singularly perturbed problems, including proofs and several examples, we refer to the surveys of Jones $([\underline{6})$ and Kaper ([7]). We note that for the singular perturbation problems studied by Fenichel only two different time scales can be derived: a slow and a fast one. In this setting there appears a third time scale which provides an intermediate problem.

EXAMPLE 1. Systems in nature, which are modeled by ordinary differential equations (ODE), often involve two or more different time scales. For instance, in biological literature we can find many examples of models which present such features. The classical Rosenzweig-MacArthur predator-prey model (see [12, [18] and [16]) that is in a rescaled form given by

$$
x^{\prime}=x\left(1-x-\frac{a y}{x+d}\right), \quad y^{\prime}=\varepsilon y\left(\frac{a x}{x+d}-1\right)
$$

serves as an example of a problem involving two different time scales. In the above system $x$ and $y$ represent the number of prey and predators, respectively, the parameter $\varepsilon>0$ is the ratio between the linear death rate of the predator and the linear growth rate of the prey, and the positive parameters $a$ and $d$ determine the impact of predation on the prey.

EXAMPLE 2. Examples of models involving three time scales are for instance found in food chain models with a third class of so-called super- or top-predators ([14, 1] and [2]) or in hormone secretion models (9]). For instance, the Rosenzweig-MacArthur model ([17]) for tritrophic food chains (as proposed by [1])

$$
\varepsilon x^{\prime}=x\left(1-x-\frac{y}{x+b_{1}}\right), \quad y^{\prime}=y\left(\frac{x}{x+b_{1}}-d_{1}-\frac{z}{y+b_{2}}\right), \quad z^{\prime}=\delta z\left(\frac{y}{y+b_{2}}-d_{2}\right)
$$


is an example of a problem involving three different time scales. It is composed of a logistic prey $x$, a Holling type II predator $y$ and a Holling type II top-predator $z$. Models with three or more time scales are also used to study neuronal behavior, in particular to explain the firing of neurons or so-called mixed mode oscillations (see [8], 13]).

Here we will study systems with three distinct time scales. These systems are in general written in the form

$$
\varepsilon x^{\prime}=f(\mathbf{x}, \varepsilon, \delta), \quad y^{\prime}=g(\mathbf{x}, \varepsilon, \delta), \quad z^{\prime}=\delta h(\mathbf{x}, \varepsilon, \delta),
$$

where $\mathbf{x}=(x, y, z) \in \mathbb{R}^{n} \times \mathbb{R}^{m} \times \mathbb{R}^{p}, \varepsilon$ and $\delta$ are two independent small parameters $(0<\varepsilon, \delta \ll 1)$, and $f, g, h$ are $C^{r}$ functions, where $r$ is big enough for our purposes. Now, in system (4) three different time scales can be derived: a slow time scale $t$, an intermediate time scale $\tau_{1}:=\frac{t}{\delta}$ and a fast time scale $\tau_{2}:=\frac{\tau_{1}}{\varepsilon}$.

Note that the model (3) is a system of the form (4). Some authors studied the Rosenzweig-MacArthur model (31) applying geometric singular perturbation techniques; see for instance [5], [1, 2] and [14. Such techniques have also been used to study other problems in population dynamics, such as competitive co-existence [15] and insect outbreaks in forest ecosystems [11].

In this paper we develop a mathematical theory in order to study systems (4). Our main goal is to build a theory, inspired by the one given by Fenichel in [3], for systems involving three different time scales. Finally, we apply this new approach to study nonsmooth dynamical systems with self-intersecting switching variety.

The paper is organized as follows. In Section 2 we state the main results involving system (4). In Theorems A and B we study the persistence and the stability properties of compact normally hyperbolic invariant sets of the reduced problem (see system (8)). The proofs these theorems appear in Section 4. In Section 3 we give some further examples where Theorems A and B are applied. In Section [5 we consider nonsmooth dynamical systems with a self-intersecting switching variety. We prove that double regularization of these systems provides a class of three time scale singular perturbation problems; see Theorem C.

2. Statement of the main results. The system (44) is written with respect to the time scale $\tau_{1}$, so it is called intermediate system. By transforming (4) to the slow and fast variables $t$ and $\tau_{2}$ we obtain, respectively, the slow system

$$
\varepsilon \delta x^{\prime}=f(\mathbf{x}, \varepsilon, \delta), \quad \delta y^{\prime}=g(\mathbf{x}, \varepsilon, \delta), \quad z^{\prime}=h(\mathbf{x}, \varepsilon, \delta),
$$

and the fast system

$$
x^{\prime}=f(\mathbf{x}, \varepsilon, \delta), \quad y^{\prime}=\varepsilon g(\mathbf{x}, \varepsilon, \delta), \quad z^{\prime}=\varepsilon \delta h(\mathbf{x}, \varepsilon, \delta) .
$$

REMARK 1. To simplify our notation, we will use the notation $x^{\prime}$ to indicate the derivative with respect to the three time scales. More specifically, for the systems (4), (5) and (6),$x^{\prime}$ indicates the derivatives $\frac{d x}{d \tau_{1}}, \frac{d x}{d t}$ and $\frac{d x}{d \tau_{2}}$, respectively.

Note that, for $\varepsilon, \delta \neq 0$, systems (41), (5) and (6) are equivalent. By setting $\varepsilon=\delta=0$ in (44), (5) and in (6) we obtain three systems with dynamics essentially different: the 
intermediate problem

$$
0=f(\mathbf{x}, 0,0), \quad y^{\prime}=g(\mathbf{x}, 0,0), \quad z^{\prime}=0,
$$

the reduced problem

$$
0=f(\mathbf{x}, 0,0), \quad 0=g(\mathbf{x}, 0,0), \quad z^{\prime}=h(\mathbf{x}, 0,0),
$$

and the layer problem

$$
x^{\prime}=f(\mathbf{x}, 0,0), \quad y^{\prime}=0, \quad z^{\prime}=0 .
$$

For each $\varepsilon$ and $\delta$, consider the following sets:

$$
\mathcal{S}_{1}^{\delta}=\left\{\mathbf{x} \in \mathbb{R}^{n+m+p}: f(\mathbf{x}, 0, \delta)=0\right\}
$$

and

$$
\mathcal{S}_{2}^{\varepsilon}=\left\{\mathbf{x} \in \mathbb{R}^{n+m+p}: f(\mathbf{x}, \varepsilon, 0)=g(\mathbf{x}, \varepsilon, 0)=0\right\} .
$$

Note that the intermediate and reduced problems (7) and (8) are dynamical systems defined on $\mathcal{S}_{1}^{0}$ and $\mathcal{S}_{2}^{0}$, respectively. On the other hand $\mathcal{S}_{1}^{0}$ is a manifold of singular points for (9). In what follows we refer to $\mathcal{S}_{1}^{0}$ and $\mathcal{S}_{2}^{0}$ as the intermediate and slow manifolds, respectively. The reason for these names is that on $\mathcal{S}_{1}^{0}$ the intermediate time scale is dominating and on $\mathcal{S}_{2}^{0}$ the slow time scale predominates.

Following the ideas of the geometric singular perturbation theory [3], our goal will be to prove that one can obtain information on the dynamics of system (4), for small values of $\varepsilon$ and $\delta$, by suitably combining the dynamics of the three limit problems (7), (8) and (9).

Four other systems will also play an important role in our analysis of system (44). By setting $\varepsilon=0$ in (4) (or in (5) ) and in (6) while keeping $\delta$ fixed but nonzero, we obtain the $\delta$-intermediate problem

$$
0=f(\mathbf{x}, 0, \delta), \quad y^{\prime}=g(\mathbf{x}, 0, \delta), \quad z^{\prime}=\delta h(\mathbf{x}, 0, \delta)
$$

and the $\delta$-layer problem

$$
x^{\prime}=f(\mathbf{x}, 0, \delta), \quad y^{\prime}=0, \quad z^{\prime}=0 .
$$

By setting $\delta=0$ in (4) (or in (6) ) and in (5) while keeping $\varepsilon$ fixed but nonzero, we obtain the $\varepsilon$-intermediate problem

$$
\varepsilon x^{\prime}=f(\mathbf{x}, \varepsilon, 0), \quad y^{\prime}=g(\mathbf{x}, \varepsilon, 0), \quad z^{\prime}=0
$$

and the $\varepsilon-$ reduced problem

$$
0=f(\mathbf{x}, \varepsilon, 0), \quad 0=g(\mathbf{x}, \varepsilon, 0), \quad z^{\prime}=h(\mathbf{x}, \varepsilon, 0) .
$$

Note that when both $\varepsilon, \delta \rightarrow 0$, the two $\delta, \varepsilon$-intermediate problems (10) and (12) become the same limit problem (77). The problems (10) and (13) are dynamical systems defined on the manifolds $\mathcal{S}_{1}^{\delta}$ and $\mathcal{S}_{2}^{\varepsilon}$, respectively. On the other hand, $\mathcal{S}_{1}^{\delta}$ and $\mathcal{S}_{2}^{\varepsilon}$ are sets of singular points for the problems (11) and (12), respectively. 


\begin{tabular}{|c|c|c|}
\hline problem & equation & label \\
\hline intermediate system & $\begin{array}{c}\varepsilon x^{\prime}=f(\mathbf{x}, \varepsilon, \delta) \\
y^{\prime}=g(\mathbf{x}, \varepsilon, \delta) \\
z^{\prime}=\delta h(\mathbf{x}, \varepsilon, \delta)\end{array}$ & (4) \\
\hline slow system & $\begin{array}{c}\varepsilon \delta x^{\prime}=f(\mathbf{x}, \varepsilon, \delta) \\
\delta y^{\prime}=g(\mathbf{x}, \varepsilon, \delta) \\
z^{\prime}=h(\mathbf{x}, \varepsilon, \delta)\end{array}$ & $(5)$ \\
\hline fast system & $\begin{array}{c}x^{\prime}=f(\mathbf{x}, \varepsilon, \delta) \\
y^{\prime}=\varepsilon g(\mathbf{x}, \varepsilon, \delta) \\
z^{\prime}=\varepsilon \delta h(\mathbf{x}, \varepsilon, \delta)\end{array}$ & (6) \\
\hline intermediate problem & $\begin{array}{c}0=f(\mathbf{x}, 0,0) \\
y^{\prime}=g(\mathbf{x}, 0,0) \\
z^{\prime}=0\end{array}$ & (7) \\
\hline reduced problem & $\begin{aligned} 0 & =f(\mathbf{x}, 0,0) \\
0 & =g(\mathbf{x}, 0,0) \\
z^{\prime} & =h(\mathbf{x}, 0,0)\end{aligned}$ & (8) \\
\hline layer problem & $\begin{array}{c}x^{\prime}=f(\mathbf{x}, 0,0) \\
y^{\prime}=0 \\
z^{\prime}=0\end{array}$ & (9) \\
\hline$\delta$-intermediate & $\begin{array}{c}0=f(\mathbf{x}, 0, \delta) \\
y^{\prime}=g(\mathbf{x}, 0, \delta) \\
z^{\prime}=\delta h(\mathbf{x}, 0, \delta)\end{array}$ & $(10)$ \\
\hline$\delta$-layer & $\begin{array}{c}x^{\prime}=f(\mathbf{x}, 0, \delta) \\
y^{\prime}=0 \\
z^{\prime}=0\end{array}$ & (11) \\
\hline$\varepsilon$-intermediate & $\begin{array}{c}\varepsilon x^{\prime}=f(\mathbf{x}, \varepsilon, 0) \\
y^{\prime}=g(\mathbf{x}, \varepsilon, 0) \\
z^{\prime}=0\end{array}$ & $(12)$ \\
\hline$\varepsilon$-reduced & $\begin{aligned} 0 & =f(\mathbf{x}, \varepsilon, 0) \\
0 & =g(\mathbf{x}, \varepsilon, 0) \\
z^{\prime} & =h(\mathbf{x}, \varepsilon, 0)\end{aligned}$ & $(13)$ \\
\hline
\end{tabular}

TABLE 1. Summary of equations which appear in three time scale singular perturbation problems.

Definition 2.1. We say that system (44) is normally hyperbolic at $\mathbf{x}_{0} \in \mathcal{S}_{2}^{0}$ if the real parts of the eigenvalues of the Jacobian matrix

$$
\left(\begin{array}{c}
D_{1,2} f\left(\mathbf{x}_{0}, 0,0\right) \\
D_{1,2} g\left(\mathbf{x}_{0}, 0,0\right)
\end{array}\right)
$$


are nonzero. We say that system (4) is $\delta$-normally hyperbolic at $\mathbf{x}_{0} \in \mathcal{S}_{1}^{\delta}$ if the real parts of the eigenvalues of the Jacobian $D_{1} f\left(\mathbf{x}_{0}, 0, \delta\right)$ are nonzero.

Now we are in a position to state our main results.

Theorem A. Consider the $C^{r}$ family (44). Let $\mathcal{N} \subseteq \mathcal{S}_{2}^{0}$ be a $j$-dimensional compact normally hyperbolic invariant manifold of the reduced problem (8). Then there are $\varepsilon_{1}>0$ and $\delta_{1}>0$ and a $C^{r-1}$ family of manifolds $\left\{\mathcal{N}_{\delta}^{\varepsilon}: \delta \in\left(0, \delta_{1}\right), \varepsilon \in\left(0, \varepsilon_{1}\right)\right\}$ such that $\mathcal{N}_{0}^{0}=\mathcal{N}$ and $\mathcal{N}_{\delta}^{\varepsilon}$ is a hyperbolic invariant manifold of (4).

Consider system (10) supplemented by the trivial equation $\delta^{\prime}=0$ :

$$
0=f(\mathbf{x}, 0, \delta), \quad y^{\prime}=g(\mathbf{x}, 0, \delta), \quad z^{\prime}=\delta h(\mathbf{x}, 0, \delta), \quad \delta^{\prime}=0 .
$$

Let $G(\mathbf{x}, \delta):=(g(\mathbf{x}, 0, \delta), \delta h(\mathbf{x}, 0, \delta), 0)$ be the vector field defined by (14). Assume that the linearization of $G$ at points $(\mathbf{x}, 0)$, such that $\mathbf{x} \in \mathcal{S}_{2}^{0}$, has $k^{s}$ eigenvalues with negative real part and $k^{u}$ eigenvalues with positive real part. The corresponding stable and unstable eigenspaces have dimensions $k^{s}$ and $k^{u}$, respectively.

Similarly, consider system (6) supplemented by the trivial equation $\varepsilon^{\prime}=0$ :

$$
x^{\prime}=f(\mathbf{x}, \varepsilon, \delta), \quad y^{\prime}=\varepsilon g(\mathbf{x}, \varepsilon, \delta), \quad z^{\prime}=\varepsilon \delta h(\mathbf{x}, \varepsilon, \delta), \quad \varepsilon^{\prime}=0 .
$$

Let $H(\mathbf{x}, \varepsilon, \delta):=(f(\mathbf{x}, \varepsilon, \delta), \varepsilon g(\mathbf{x}, \varepsilon, \delta), \varepsilon \delta h(\mathbf{x}, \varepsilon, \delta), 0)$ be the vector field defined by (15). Assume that the linearization of $H$ at points $(\mathbf{x}, 0, \delta)$, such that $\mathbf{x} \in \mathcal{S}_{1}^{\delta}$, has $l^{s}$ and $l^{u}$ eigenvalues with negative and positive real parts, so that the corresponding stable and unstable eigenspaces have dimensions $l^{s}$ and $l^{u}$, respectively.

Theorem B. Under the hypotheses of Theorem A, suppose that $\mathcal{N}$ has a $\left(j+j^{s}\right)$ dimensional local stable manifold $W^{s}$ and a $\left(j+j^{u}\right)$-dimensional local unstable manifold $W^{u}$. Then there are $\varepsilon_{1}>0$ and $\delta_{1}>0$ and $C^{r-1}$ families of $\left(j+j^{s}+k^{s}+l^{s}\right)$ dimensional and $\left(j+j^{u}+k^{u}+l^{u}\right)$-dimensional manifolds $\left\{\mathcal{W}_{\delta, \varepsilon}^{s}: \delta \in\left(0, \delta_{1}\right), \varepsilon \in\left(0, \varepsilon_{1}\right)\right\}$ and $\left\{\mathcal{W}_{\delta, \varepsilon}^{u}: \delta \in\left(0, \delta_{1}\right), \varepsilon \in\left(0, \varepsilon_{1}\right)\right\}$ such that for $\delta, \varepsilon>0$ the manifolds $\left\{\mathcal{W}_{\delta, \varepsilon}^{s}\right\}$ and $\left\{\mathcal{W}_{\delta, \varepsilon}^{u}\right\}$ are local stable and unstable manifolds of $\mathcal{N}_{\delta}^{\varepsilon}$, respectively.

Theorems A and B are proved in Section 4. They are illustrated by the example below. Let us start with a simplest possible example of a system of the form (4).

EXAMPLE 3. Consider the following 3-dimensional system:

$$
\varepsilon x^{\prime}=x-\varepsilon+\delta, \quad y^{\prime}=-y+\varepsilon+\delta, \quad z^{\prime}=\delta z .
$$

The intermediate and slow manifolds $\mathcal{S}_{1}^{0}$ and $\mathcal{S}_{2}^{0}$ are given, respectively, by $\mathcal{S}_{1}^{0}=\{(x, y, z) \in$ $\left.\mathbb{R}^{3}: x=0\right\}$ and $\mathcal{S}_{2}^{0}=\left\{(x, y, z) \in \mathbb{R}^{3}: x=y=0\right\}$. On $\mathcal{S}_{1}^{0}$ we have defined the intermediate problem

$$
0=x, \quad y^{\prime}=-y, \quad z^{\prime}=0,
$$

and on $\mathcal{S}_{2}^{0}$ we have defined the reduced problem

$$
0=x, \quad 0=y, \quad z^{\prime}=z .
$$

Moreover, the layer problem is given by

$$
x^{\prime}=x, \quad y^{\prime}=0, \quad z^{\prime}=0 .
$$



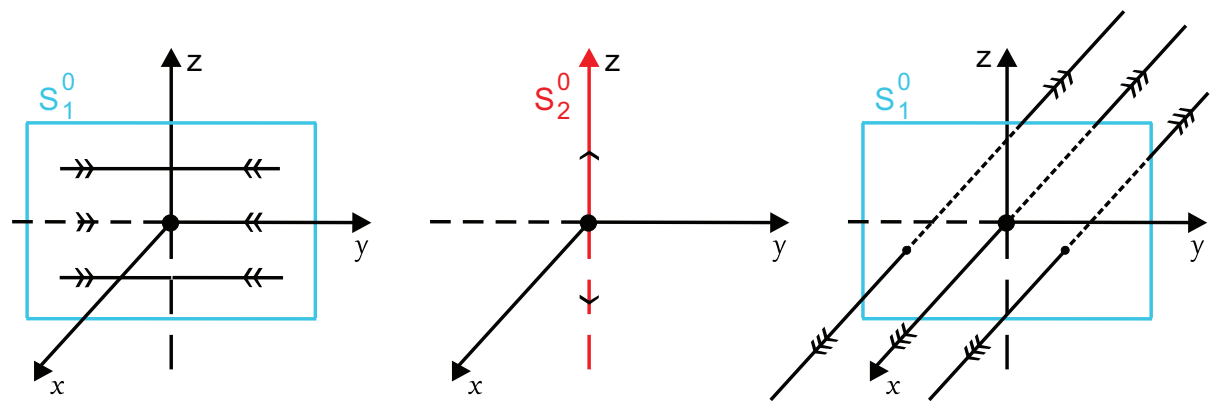

FIG. 1. Phase portraits of systems (17), 18 and (19), respectively.

Figure 1 illustrates the phase portraits of the intermediate, reduced and layer problems, respectively.

By using the notation given in Theorem $\mathrm{B}$, note that we have $j=0, j^{s}=0, j^{u}=1$, $k^{s}=1, k^{u}=0, l^{s}=0$ e $l^{u}=1$. We can then apply Theorems $\mathrm{A}$ and $\mathrm{B}$ at the normally hyperbolic singular point $\mathcal{N}=(0,0,0)$ of (18). Applying Theorem A, we obtain for small nonzero $\delta, \varepsilon$, a family $\mathcal{N}_{\delta}^{\varepsilon}$ of hyperbolic singular points of (16). In fact, the family $\mathcal{N}_{\delta}^{\varepsilon}$ of singular points is given by $(\varepsilon-\delta, \varepsilon+\delta, 0)$. Applying Theorem $\mathrm{B}$, we can conclude that each singular point $\mathcal{N}_{\delta}^{\varepsilon}$ has a 1 -dimensional local stable manifold $\mathcal{W}_{\delta, \varepsilon}^{s}$ and a 2 -dimensional local unstable manifold $\mathcal{W}_{\delta, \varepsilon}^{u}$.

3. Further examples. In this section we give some further examples where Theorems $\mathrm{A}$ and $\mathrm{B}$ are applied. In the next example we study the dynamics of a biological model.

EXAmple 4. Consider the Rosenzweig-MacArthur model ([17]) for tritrophic food chains (as proposed by [1])

$$
\begin{aligned}
\varepsilon x^{\prime} & =x\left(1-x-\frac{y}{x+b_{1}}\right)=x f(x, y), \\
y^{\prime} & =y\left(\frac{x}{x+b_{1}}-d_{1}-\frac{z}{y+b_{2}}\right)=y g(x, y, z), \\
z^{\prime} & =\delta z\left(\frac{y}{y+b_{2}}-d_{2}\right)=\delta z h(y),
\end{aligned}
$$

where $x, y$ and $z$ are 1 -dimensional variables which represent a logistic prey, a Holling type II predator and a Holling type II top-predator, respectively. All parameters $b_{1}, b_{2}$, $d_{1}$ and $d_{2}$ are assumed to be positive and less than 1 , i.e., $0<b_{1}, b_{2}, d_{1}, d_{2}<1$. We note that all discussions below are restricted to the first octant, i.e., $x \geq 0, y \geq 0$ and $z \geq 0$.

The intermediate and slow manifolds $\mathcal{S}_{1}^{0}$ and $\mathcal{S}_{2}^{0}$ are given, respectively, by $\mathcal{S}_{1}^{0}=$ $\{x f(x, y)=0\}=\{(x, y, z): x=0\} \cup\left\{(x, y, z): y=(1-x)\left(b_{1}+x\right)\right\}=M_{1} \cup M_{2}$ and 

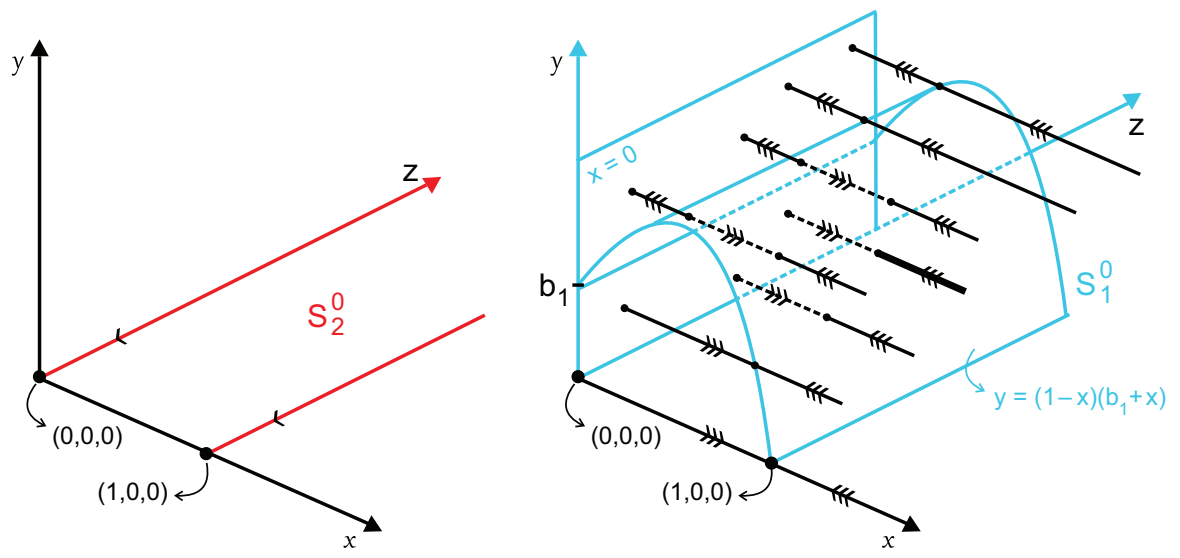

FIG. 2. Phase portraits of systems (23) and (24), respectively.

$\mathcal{S}_{2}^{0}=\{x f(x, y)=y g(x, y, z)=0\}=\{(x, y, z): x=y=0\} \cup\{(x, y, z): x=1, y=0\}=$ $M_{3} \cup M_{4}$.

The intermediate problem is a dynamical system defined on $\mathcal{S}_{1}^{0}=M_{1} \cup M_{2}$. On $M_{1}$ it is given by

$$
y^{\prime}=y\left(-d_{1}-\frac{z}{y+b_{2}}\right), \quad z^{\prime}=0,
$$

and on $M_{2}$ it becomes

$$
y^{\prime}=y\left(\frac{x}{x+b_{1}}-d_{1}-\frac{z}{y+b_{2}}\right), \quad z^{\prime}=0 .
$$

The reduced problem is a dynamical system defined on $\mathcal{S}_{2}^{0}=M_{3} \cup M_{4}$. On both $M_{3}$ and $M_{4}$ it is given by

$$
z^{\prime}=-d_{2} z
$$

The layer problem is given by

$$
x^{\prime}=x\left(1-x-\frac{y}{x+b_{1}}\right), \quad y^{\prime}=0, \quad z^{\prime}=0 .
$$

Figure 2 illustrates the phase portraits of the reduced and layer problems, respectively. Figure 3 illustrates the phase portraits of systems (21) and (22), respectively. For the phase portrait of (22) we are assuming that $1 /\left(1+b_{1}\right)>d_{1}$.

Note that $\mathcal{N}=(0,0,0)$ and $\mathcal{M}=(1,0,0)$ are singular points of (23). Moreover, according to Definition 2.1. system (20) is normally hyperbolic at $\mathcal{N}$ and $\mathcal{M}$ (for the point $\mathcal{M}$ we are supposing that $\left.d_{1} \neq 1 /\left(1+b_{1}\right)\right)$. Applying Theorem A, we obtain for small nonzero $\delta, \varepsilon$, families $\mathcal{N}_{\delta}^{\varepsilon}$ and $\mathcal{M}_{\delta}^{\varepsilon}$ of hyperbolic singular points of (20). In fact, the persistent singular points $\mathcal{N}_{\delta}^{\varepsilon}$ and $\mathcal{M}_{\delta}^{\varepsilon}$ are given by $(0,0,0)$ and $(1,0,0)$, respectively.

By using the notation given in Theorem B, we have that: for the point $\mathcal{N}, j=0$, $j^{s}=1, j^{u}=0, k^{s}=1, k^{u}=0, l^{s}=0$ e $l^{u}=1$, and for the point $\mathcal{M}, j=0, j^{s}=1$, $j^{u}=0, k^{s}=0, k^{u}=1, l^{s}=1$ e $l^{u}=0$. Applying Theorem B, we can conclude that each 

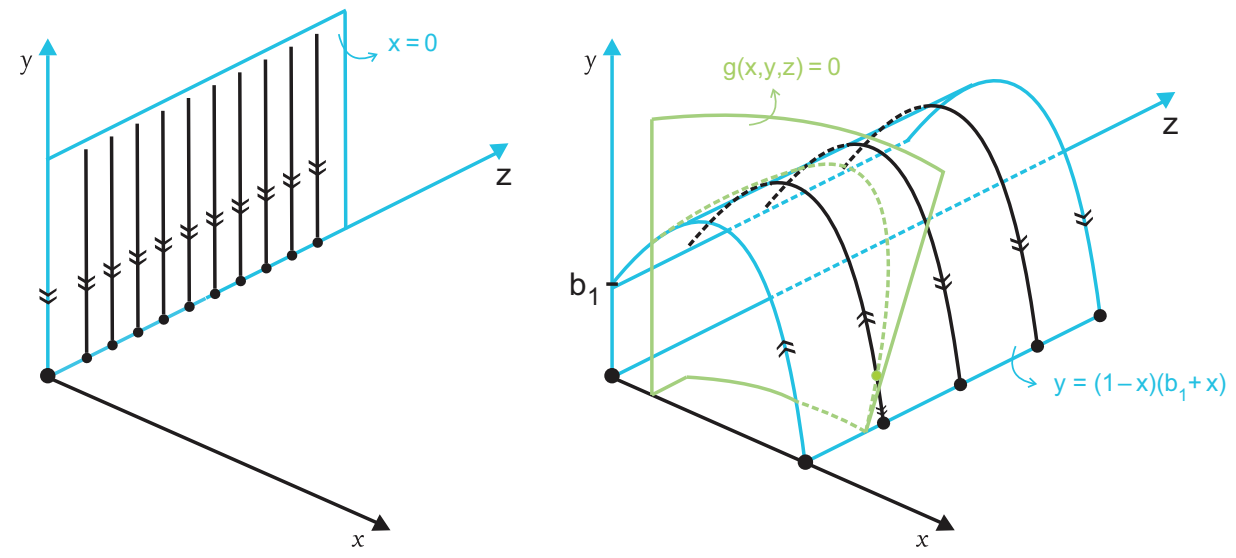

FIG. 3. Phase portraits of systems (21) and (22), respectively.

singular point $\mathcal{N}_{\delta}^{\varepsilon}$ has a 2 -dimensional local stable manifold $\mathcal{W}_{\delta, \varepsilon}^{s}$ and a 1 -dimensional local unstable manifold $\mathcal{W}_{\delta, \varepsilon}^{u}$. Moreover, each singular point $\mathcal{M}_{\delta}^{\varepsilon}$ has a 2-dimensional local stable manifold $\overline{\mathcal{W}}_{\delta, \varepsilon}^{s}$ and a 1-dimensional local unstable manifold $\overline{\mathcal{W}}_{\delta, \varepsilon}^{u}$.

EXAmple 5. Consider the following 4-dimensional system:

$$
\begin{aligned}
\varepsilon x^{\prime} & =x-z_{1}+\delta+\varepsilon=f\left(x, z_{1}, \delta, \varepsilon\right), \\
y^{\prime} & =-y-z_{2}+\delta-\varepsilon=g\left(y, z_{2}, \delta, \varepsilon\right), \\
z_{1}^{\prime} & =\delta h_{1}\left(x, z_{1}, z_{2}\right) \\
z_{2}^{\prime} & =\delta h_{2}\left(y, z_{1}, z_{2}\right)
\end{aligned}
$$

where

$$
h_{1}\left(x, z_{1}, z_{2}\right)=-z_{2}-z_{1}\left(-1+z_{1}^{2}+z_{2}^{2}\right)+\left(x-z_{1}\right)^{2}
$$

and

$$
h_{2}\left(y, z_{1}, z_{2}\right)=z_{1}-z_{2}\left(-1+z_{1}^{2}+z_{2}^{2}\right)-\left(y+z_{2}\right)^{2} .
$$

The intermediate and slow manifolds $\mathcal{S}_{1}^{0}$ and $\mathcal{S}_{2}^{0}$ are given, respectively, by $\mathcal{S}_{1}^{0}=$ $\left\{\left(z_{1}, y, z_{1}, z_{2}\right) \in \mathbb{R}^{4}: y, z_{1}, z_{2} \in \mathbb{R}\right\}$ and $\mathcal{S}_{2}^{0}=\left\{\left(z_{1},-z_{2}, z_{1}, z_{2}\right) \in \mathbb{R}^{4}: z_{1}, z_{2} \in \mathbb{R}\right\}$. Note that $\mathcal{S}_{1}^{0}$ and $\mathcal{S}_{2}^{0}$ are manifolds of dimension 3 and 2 , respectively.

On $\mathcal{S}_{1}^{0}$ we have defined the intermediate problem

$$
x=z_{1}, \quad y^{\prime}=-y-z_{2}, \quad z_{1}^{\prime}=0, \quad z_{2}^{\prime}=0,
$$

and on $\mathcal{S}_{2}^{0}$ we have defined the reduced problem

$$
x=z_{1}, \quad y=-z_{2}, \quad z_{1}^{\prime}=-z_{2}-z_{1}\left(-1+z_{1}^{2}+z_{2}^{2}\right), \quad z_{2}^{\prime}=z_{1}-z_{2}\left(-1+z_{1}^{2}+z_{2}^{2}\right) .
$$

Moreover, the layer problem is given by

$$
x^{\prime}=x-z_{1}, \quad y^{\prime}=0, \quad z_{1}^{\prime}=0, \quad z_{2}^{\prime}=0 .
$$

For the phase portrait of the reduced problem we can use polar coordinates $z_{1}=r \cos \theta$ and $z_{2}=r \sin \theta$. Using these coordinates it is easy to see that system (27) presents a singular point $\mathcal{P}$ at the origin and a stable limit cycle $\Gamma$, as shown Figure 4 . 


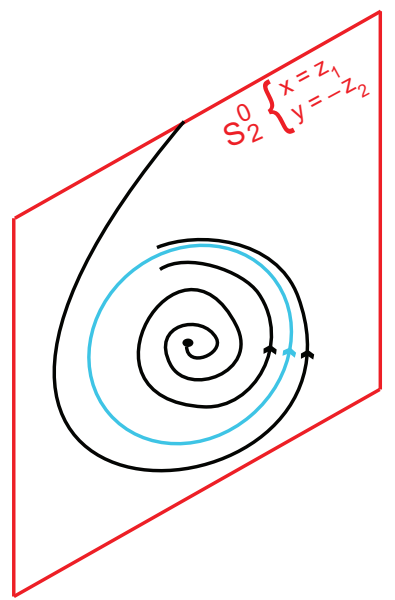

FIG. 4. Phase portrait of system (27).

According to Definition 2.1, all points of the slow manifold are normally hyperbolic. Applying Theorem A, we obtain for small nonzero $\delta, \varepsilon$, families $\mathcal{P}_{\delta}^{\varepsilon}$ and $\Gamma_{\delta}^{\varepsilon}$ of hyperbolic singular points and limit cycles of (25), respectively, such that $\mathcal{P}_{0}^{0}=\mathcal{P}$ and $\Gamma_{0}^{0}=\Gamma$. Вy using the notation given in Theorem $\mathrm{B}$, we have that: for the point $\mathcal{P}, j=0, j^{s}=0$, $j^{u}=2, k^{s}=1, k^{u}=0, l^{s}=0$ e $l^{u}=1$, and for the limit cycle $\Gamma, j=1, j^{s}=1, j^{u}=-1$, $k^{s}=1, k^{u}=0, l^{s}=0$ e $l^{u}=1$. In agreement with Theorem $\mathrm{B}$, each singular point $\mathcal{P}_{\delta}^{\varepsilon}$ has a 1 -dimensional local stable manifold $\mathcal{P}_{\delta, \varepsilon}^{s}$ and a 3 -dimensional local unstable manifold $\mathcal{P}_{\delta, \varepsilon}^{u}$. Each limit cycle $\Gamma_{\delta}^{\varepsilon}$ has a 3 -dimensional local stable manifold $\Gamma_{\delta, \varepsilon}^{s}$ and a 1-dimensional local unstable manifold $\Gamma_{\delta, \varepsilon}^{u}$.

4. Proofs of Theorems A and B. In this section we prove Theorems A and B.

First we prove Theorem A.

Proof of Theorem A. Firstly we use Fenichel's first theorem to study the persistence of $\mathcal{N}$ under $\delta$-perturbations of the system (10). Fenichel's first theorem states that the compact normally hyperbolic invariant manifold $\mathcal{N}$ of the reduced problem (8) persists, for $\delta \neq 0$ small, as an invariant manifold $\mathcal{N}_{\delta}$ for system (10). More precisely, there exists $\delta_{1}>0$ and a $C^{r-1}$ family of manifolds $\left\{\mathcal{N}_{\delta}: \delta \in\left(-\delta_{1}, \delta_{1}\right)\right\}$ such that $\mathcal{N}_{0}=\mathcal{N}$ and $\mathcal{N}_{\delta}$ is a hyperbolic invariant manifold of (10). Now, for each $\delta$ fixed, we again use the Fenichel's theory to study the persistence of $\mathcal{N}_{\delta}$ under $\varepsilon$-perturbations of system (41). Note that system (10) corresponds to the reduced problem associated to system (44). Fenichel's first theorem says that the compact $\delta$-normally hyperbolic invariant manifold $\mathcal{N}_{\delta}$ of (10) persists, for $\varepsilon \neq 0$ sufficiently small, for system (4); that is, there exists $\varepsilon_{1}>0$ and a $C^{r-1}$ family of manifolds $\left\{\mathcal{N}_{\delta}^{\varepsilon}: \varepsilon \in\left(-\varepsilon_{1}, \varepsilon_{1}\right)\right\}$ such that $\mathcal{N}_{\delta}^{0}=\mathcal{N}_{\delta}$ and $\mathcal{N}_{\delta}^{\varepsilon}$ is a hyperbolic invariant manifold of (4). This completes the proof of Theorem A.

Now we prove Theorem B. 
Proof of Theorem B. Fenichel's second theorem says that, for small nonzero $\delta$, the invariant manifold $\mathcal{N}_{\delta}$ of (10) has a $\left(j+j^{s}+k^{s}\right)$-dimensional local stable manifold $\mathcal{W}_{\delta}^{s}$ and a $\left(j+j^{u}+k^{u}\right)$-dimensional local unstable manifold $\mathcal{W}_{\delta}^{u}$. Now, for each $\delta$ fixed, Fenichel's second theorem also states that, for $\varepsilon \neq 0$ sufficiently small, the invariant manifold $\mathcal{N}_{\delta}^{\varepsilon}$ of (4) has a $\left(j+j^{s}+k^{s}+l^{s}\right)$-dimensional local stable manifold $\mathcal{W}_{\delta, \varepsilon}^{s}$ and a $\left(j+j^{u}+k^{u}+l^{u}\right)$-dimensional local unstable manifold $\mathcal{W}_{\delta}^{u}$. This completes the proof of Theorem B.

5. Application: Non-smooth dynamical systems. The study of non-smooth dynamical systems has, in recent years, established an important frontier between mathematics, physics and engineering. For a survey on qualitative aspects of such systems we refer to [20] and the references therein.

Consider $\Sigma=\Sigma_{1} \cup \Sigma_{2} \subset \mathbb{R}^{3}$, where $\Sigma_{1}$ and $\Sigma_{2}$ are codimension one sub-manifolds of $\mathbb{R}^{3}$ with $0 \in \Sigma_{1} \cap \Sigma_{2}$, that are in general position. Around $0 \in \mathbb{R}^{3}$, we have that $\Sigma_{1} \cup \Sigma_{2}$ separates $\mathbb{R}^{3}$ into four open quadrants: $Q_{1}, \ldots, Q_{4}$. In our approach $\Sigma$ will be the discontinuity boundary of our systems also named switching variety.

Let $X_{i}, i=1, \ldots, 4$, be $C^{\kappa}$ vector fields, with $\kappa \geqslant 1, \kappa=\infty$ or $\kappa=\omega$, defined on $\mathbb{R}^{3}$. Consider the discontinuous differential system

$$
\rho^{\prime}=X(\rho)=X_{i}(\rho), \quad \rho \in Q_{i}, \quad i=1, \ldots, 4 .
$$

We will denote these systems by $X=\left(X_{1}, \ldots, X_{4}\right) \in \Omega^{\kappa}$ and the intersection $\overline{Q_{i}} \cap \overline{Q_{j}}$ by $\Sigma_{i j}$. We consider local coordinates $(x, y, z)$ such that $\Sigma_{1}=\Sigma_{14} \cup \Sigma_{23}=\{z=0\}$, $\Sigma_{2}=\Sigma_{12} \cup \Sigma_{34}=\{y=0\}$ and

$$
\begin{array}{ll}
Q_{1}=\{y>0, z>0\}, & Q_{2}=\{y<0, z>0\}, \\
Q_{3}=\{y<0, z<0\}, & Q_{4}=\{y>0, z<0\} .
\end{array}
$$

Denote by $\Sigma^{*}=\Sigma \backslash\left(\Sigma_{1} \cap \Sigma_{2}\right)$ the regular part of $\Sigma$. In $\Sigma^{*}$ the definition of an orbitsolution obeys, whenever is possible, the Filippov convention (see [4]). Consider $Q_{i}$ and $Q_{j}, i \neq j$, having a common boundary. According to this convention there may exist generically a sliding region $\Sigma^{s l} \subset \Sigma^{*}$ such that any orbit which meets $\Sigma^{s l}$ remains tangent to $\Sigma^{*}$ for positive time. This region is the part of $\Sigma^{*}$ on which $X_{i}$ and $X_{j}$ point inward to $\Sigma^{*}$. Analogously there may exist generically an escaping region $\Sigma^{e s} \subset \Sigma^{*}$ such that any orbit which meets $\Sigma^{e s}$ remains tangent to $\Sigma^{*}$ for negative time.

On $\Sigma^{s l} \cup \Sigma^{e s}$ the flow slides on $\Sigma^{*}$; the flow follows a well defined vector field $X^{\Sigma}$ called a sliding vector field. The sewing region $\Sigma^{s w} \subset \Sigma^{*}$ is the part of $\Sigma^{*}$ where the flow crosses $\Sigma^{*}$. The boundary between the three regions is the locus of points where the vector field is tangent to $\Sigma^{*}$. One of our concerns is to know when a sliding flow in $\Sigma^{*}$ can be continued until the intersection $\Sigma_{1} \cap \Sigma_{2}$.

The sliding vector field $X^{\Sigma}$ is defined at $q \in \Sigma_{k j} \cap\left(\Sigma^{s l} \cup \Sigma^{e s}\right)$ by $X^{\Sigma}(q)=m-q$ with $m$ being the point where the segment joining $q+X_{k}(q)$ and $q+X_{j}(q)$ cuts $\Sigma_{k j}$.

We denote

$$
\widehat{\Sigma_{1}}=\left\{(\theta, x, y) ; \theta \in(0, \pi),(x, y) \in \mathbb{R}^{2}\right\}
$$


and

$$
\widehat{\Sigma_{2}}=\left\{(\psi, x, z) ; \psi \in(0, \pi),(x, z) \in \mathbb{R}^{2}\right\} .
$$

In a previous work [10] we proof the following theorem.

Theorem C. Consider $X=\left(X_{1}, \ldots, X_{4}\right) \in \Omega^{\kappa}$. There exist slow-fast systems

$$
\begin{gathered}
\theta^{\prime}=\alpha_{i}(r, \theta, x, y), \quad x^{\prime}=r \beta_{i}(r, \theta, x, y), \quad y^{\prime}=r \sigma_{i}(r, \theta, x, y), \quad i=1,2 ; \\
\psi^{\prime}=\gamma_{i}(u, \psi, x, z), \quad x^{\prime}=u \delta_{i}(u, \psi, x, z), \quad z^{\prime}=u \nu_{i}(u, \psi, x, z), \quad i=1,2
\end{gathered}
$$

and a three time scale singular perturbation problem

$$
u \psi^{\prime}=r \zeta(r, u, \theta, \psi, x), \quad \theta^{\prime}=\xi(r, u, \theta, \psi, x), \quad x^{\prime}=r \phi(r, u, \theta, \psi, x)
$$

with $r, u \geq 0, \theta, \psi \in[0, \pi],(x, y, z) \in \mathbb{R}^{3}, \alpha_{i}, \beta_{i}, \sigma_{i}, \gamma_{i}, \delta_{i}, \nu_{i}, \zeta, \xi, \phi \in C^{\kappa}$ for $i=1,2$, satisfying:

(a) The sliding region $\left(\Sigma^{s l} \cup \Sigma^{e s}\right) \cap \Sigma_{1}$ is homeomorphic to the slow manifold $\left\{\alpha_{i}(0, \theta, x, y)=0\right\}$ of (30), with $i=1$ at $\widehat{\Sigma_{1}} \cap\{y>0\}$ and with $i=2$ at $\widehat{\Sigma_{1}} \cap\{y<0\}$. The sliding vector field $X^{\Sigma}$ is topologically equivalent to the reduced problem $\alpha_{i}(0, \theta, x, y)=0, x^{\prime}=\beta_{i}(0, \theta, x, y)$ and $y^{\prime}=\sigma_{i}(0, \theta, x, y)$.

(b) The sliding region $\left(\Sigma^{s l} \cup \Sigma^{e s}\right) \cap \Sigma_{2}$ is homeomorphic to the slow manifold $\left\{\gamma_{i}(0, \psi, x, z)=0\right\}$ of (31), with $i=1$ at $\widehat{\Sigma_{2}} \cap\{z>0\}$ and with $i=2$ at $\widehat{\Sigma_{2}} \cap\{z<0\}$. The sliding vector field $X^{\Sigma}$ is topologically equivalent to the reduced problem $\gamma_{i}(0, \psi, x, z)=0, x^{\prime}=\delta_{i}(0, \psi, x, z)$ and $z^{\prime}=\nu_{i}(0, \psi, x, z)$.

(c) System (32) is the blowing up of the regularization of systems (30) for $i=1,2$. The intermediate manifold is given by $\mathcal{S}_{1}^{0}=\{\zeta(0,0, \theta, \psi, x)=0\}$. Furthermore, the intermediate flow is the limit, for $r, u \downarrow 0$, of the trajectories of another singular perturbation expressed by

$$
r \theta^{\prime}=\xi(r, u, \theta, \psi, x), \quad x^{\prime}=\phi(r, u, \theta, \psi, x) .
$$

The slow manifold of (33) is the set on $\mathbb{R}^{\ell}$ given by

$$
\mathcal{S}_{2}^{0}=\{\xi(0,0, \theta, \psi, x)=0, \zeta(0,0, \theta, \psi, x)=0\} \subseteq \mathcal{S}_{1}^{0} .
$$

The proof of Theorem $\mathrm{C}$, for systems defined on $\mathbb{R}^{\ell}$, can be found in [10]. To make our text more complete, we reproduce the steps of the proof in the following example.

Example 6 . Consider $X=\left(X_{1}, \ldots, X_{4}\right)$, where $X_{1}=(-x,-1,-1), X_{2}=(-x, 2,-3)$, $X_{3}=(-x, 1,2)$ and $X_{4}=(-x,-3,1)$. Consider the transition function $\varphi: \mathbb{R}: \rightarrow \mathbb{R}$ given by $\varphi(s)=\frac{2}{\pi} \arctan (s)$.

The $\varphi_{y}$-regularization of $X$ is the one-parameter family given by

$$
\begin{aligned}
X_{\varepsilon}^{12} & =(1 / 2)\left[(1+\varphi(y / \varepsilon)) X_{1}+(1-\varphi(y / \varepsilon)) X_{2}\right] \\
& =\left(-x, \frac{1}{2}-\frac{3}{2} \varphi\left(\frac{y}{\varepsilon}\right),-2+\varphi\left(\frac{y}{\varepsilon}\right)\right)
\end{aligned}
$$

for $z>0, \varepsilon>0$, and

$$
\begin{aligned}
X_{\varepsilon}^{43} & =(1 / 2)\left[(1+\varphi(y / \varepsilon)) X_{4}+(1-\varphi(y / \varepsilon)) X_{3}\right] \\
& =\left(-x,-1-2 \varphi\left(\frac{y}{\varepsilon}\right), \frac{3}{2}-\frac{1}{2} \varphi\left(\frac{y}{\varepsilon}\right)\right)
\end{aligned}
$$

for $z<0, \varepsilon>0$. 
The $\varphi_{z}$-regularization of $X$ is the one-parameter family given by

$$
\begin{aligned}
X_{a}^{14} & =(1 / 2)\left[(1+\varphi(z / a)) X_{1}+(1-\varphi(z / a)) X_{4}\right] \\
& =\left(-x,-2+\varphi\left(\frac{z}{a}\right),-\varphi\left(\frac{z}{a}\right)\right)
\end{aligned}
$$

for $y>0, a>0$, and

$$
\begin{aligned}
X_{a}^{23} & =(1 / 2)\left[(1+\varphi(z / a)) X_{2}+(1-\varphi(z / a)) X_{3}\right] \\
& =\left(-x, \frac{3}{2}+\frac{1}{2} \varphi\left(\frac{z}{a}\right),-\frac{1}{2}-\frac{5}{2} \varphi\left(\frac{z}{a}\right)\right)
\end{aligned}
$$

for $y<0, a>0$.

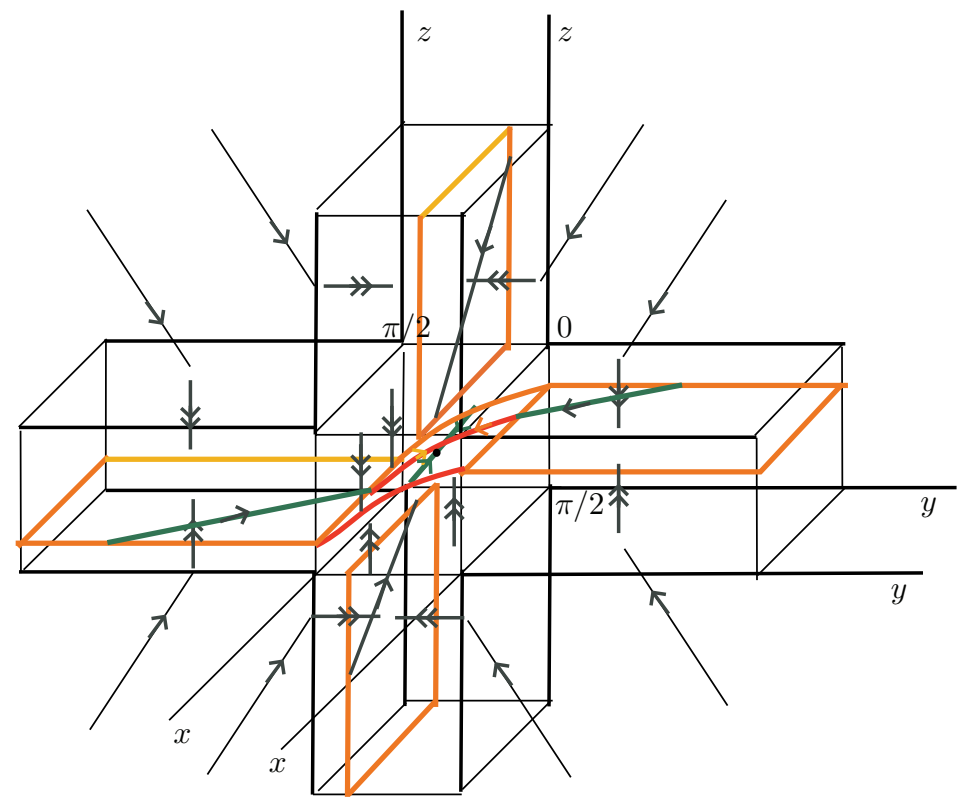

FIG. 5. A sequence of slow manifolds $\mathcal{S}_{2}^{0} \subseteq \mathcal{S}_{1}^{0}$.

Now we blow up the variables $y$ and $\varepsilon$ in $X_{\varepsilon}^{12}$ and $X_{\varepsilon}^{43}$ with $y=u \cos \psi, \varepsilon=u \sin \psi$; after we will blow up the variables $z$ and $a$ in $X_{a}^{14}$ and $X_{a}^{23}$ with $z=r \cos \theta, a=r \sin \theta$. Denoting $\lambda(s)=\varphi(\cot s), S_{\alpha}=-\sin (\alpha)$ we have:

$$
\begin{gathered}
X_{u}^{12}: \quad u \psi^{\prime}=S_{\psi}\left(\frac{1}{2}-\frac{3}{2} \lambda(\psi)\right), \quad x^{\prime}=-x, \quad z^{\prime}=-2+\lambda(\psi), \\
X_{u}^{43}: \quad u \psi^{\prime}=S_{\psi}(-1-2 \lambda(\psi)), \quad x^{\prime}=-x, \quad z^{\prime}=\frac{3}{2}-\frac{1}{2} \lambda(\psi), \\
X_{r}^{14}: \quad r \theta^{\prime}=-S_{\theta} \lambda(\theta), \quad x^{\prime}=-x, \quad y^{\prime}=-2+\lambda(\theta), \\
X_{r}^{23}: \quad r \theta^{\prime}=S_{\theta}\left(-\frac{1}{2}-\frac{5}{2} \lambda(\theta)\right), \quad x^{\prime}=-x, \quad y^{\prime}=\frac{3}{2}+\frac{1}{2} \lambda(\theta) .
\end{gathered}
$$


Rescale $X_{r}^{14}$ and $X_{r}^{23}$, perform the $\varphi_{y}$-regularization and blow up $y=u \cos \psi, \varepsilon=$ $u \sin \psi$ :

$$
\begin{aligned}
u \psi^{\prime} & =r S_{\psi}\left(-\frac{1}{4}+\frac{3}{4} \lambda(\theta)-\frac{7}{4} \lambda(\psi)+\frac{1}{4} \lambda(\theta) \lambda(\psi)\right), \\
\theta^{\prime} & =S_{\theta}\left(-\frac{1}{4}-\frac{7}{4} \lambda(\theta)+\frac{1}{4} \lambda(\psi)+\frac{3}{4} \lambda(\theta) \lambda(\psi)\right), \\
x^{\prime} & =-r x .
\end{aligned}
$$

The intermediate manifold $\mathcal{S}_{1}^{0}$ is given by $-1+3 \lambda(\theta)-7 \lambda(\psi)+\lambda(\theta) \lambda(\psi)=0$. This is a surface parameterized by $(\theta, \psi(\theta), x)$ with $\theta \in[0, \pi]$. The slow manifold $\mathcal{S}_{2}^{0}$ is represented by

$$
\left\{\begin{array}{l}
-1+3 \lambda(\theta)-7 \lambda(\psi)+\lambda(\theta) \lambda(\psi)=0, \\
-1-7 \lambda(\theta)+\lambda(\psi)+3 \lambda(\theta) \lambda(\psi)=0 .
\end{array}\right.
$$

This curve is parameterized by $\left(\theta_{0}, \psi_{0}, x\right), x \in \mathbb{R}$, with $\lambda\left(\psi_{0}\right)=\frac{11-4 \sqrt{11}}{2}$ and $\lambda\left(\theta_{0}\right)=$ $\frac{3-\sqrt{11}}{2}$. Since $x^{\prime}=-x$ the slow flow on $S_{2}^{0}$ has an attracting singular point at $\left(\theta_{0}, \psi_{0}, 0\right)$. See Figure 5 ,

Acknowledgments. The first author is supported by FAPESP grant 2013/21947-6. The second author is partially supported by CNPq-Brazil, FAPESP and FP7-PEOPLE2012-IRSES 318999 BREUDS. The third author is partially supported by FAPESP grant 2012/18780-0 and CNPq grant 300596/2009-0. All authors are supported by CAPES grant 88881.030454/2013-01 Program CSF-PVE.

\section{REFERENCES}

[1] B. Deng, Food chain chaos due to junction-fold point, Chaos 11(3) (2001), 514-525.

[2] B. Deng and G. Hines, Food chain chaos due to Shilnikov's orbit, Chaos 12 (2002), no. 3, 533-538, DOI 10.1063/1.1482255. MR1939450(2003j:92014)

[3] N. Fenichel, Geometric singular perturbation theory for ordinary differential equations, J. Differential Equations 31 (1979), no. 1, 53-98, DOI 10.1016/0022-0396(79)90152-9. MR.524817 (80m:58032)

[4] A. F. Filippov, Differential equations with discontinuous righthand sides, Mathematics and its Applications (Soviet Series), vol. 18, Kluwer Academic Publishers Group, Dordrecht, 1988. Translated from the Russian. MR.1028776 (90i:34002)

[5] G. Hek, Geometric singular perturbation theory in biological practice, J. Math. Biol. 60 (2010), no. 3, 347-386, DOI 10.1007/s00285-009-0266-7. MR2576546 (2011b:34151)

[6] C. K. R. T. Jones, Geometric singular perturbation theory, Dynamical systems (Montecatini Terme, 1994), Lecture Notes in Math., vol. 1609, Springer, Berlin, 1995, pp. 44-118, DOI 10.1007/BFb0095239. MR1374108 (97e:34105)

[7] T. J. Kaper, An introduction to geometric methods and dynamical systems theory for singular perturbation problems, (Baltimore, MD, 1998), Proc. Sympos. Appl. Math., vol. 56, Amer. Math. Soc., Providence, RI, 1999, pp. 85-131. MR1718893 (2000h:34090)

[8] M. Krupa, N. Popović, and N. Kopell, Mixed-mode oscillations in three time-scale systems: a prototypical example, SIAM J. Appl. Dyn. Syst. 7 (2008), no. 2, 361-420, DOI 10.1137/070688912. MR 2421110 (2009f:34143)

[9] W. Kunpasuruang, Y. Lenbury, and G. Hek, A nonlinear mathematical model for pulsatile discharges of luteinizing hormone mediated by hypothalamic and extra-hypothalamic pathways, Math. Models Methods Appl. Sci. 12 (2002), no. 5, 607-624, DOI 10.1142/S0218202502001817. MR.1909419 (2003k:92006)

[10] J. Llibre, P. R. da Silva, and M. A. Teixeira, Study of singularities in nonsmooth dynamical systems via singular perturbation, SIAM J. Appl. Dyn. Syst. 8 (2009), no. 1, 508-526, DOI 10.1137/080722886. MR2496766(2010a:34117) 
[11] D. Ludwig, D. D. Jones and C. S. Holling, Qualitative analysis of insect outbreak systems: the spruce budworm and forest, J. Anim. Ecol. 47 (1978), 315.

[12] R. M. May, Limit cycles in predator-prey communities. Science 177 (1972), 900-902.

[13] G. S. Medvedev and J. E. Cisternas, Multimodal regimes in a compartmental model of the dopamine neuron, Phys. D 194 (2004), no. 3-4, 333-356, DOI 10.1016/j.physd.2004.02.006. MR2075659

[14] S. Muratori and S. Rinaldi, Low- and high-frequency oscillations in three-dimensional food chain systems, SIAM J. Appl. Math. 52 (1992), no. 6, 1688-1706, DOI 10.1137/0152097. MR1191357 (93m:92024)

[15] S. Muratori and S. Rinaldi, Remarks on competitive coexistence, SIAM J. Appl. Math. 49 (1989), no. 5, 1462-1472, DOI 10.1137/0149088. MR1015073 (90k:92050)

[16] S. Rinaldi and S. Muratori, Slow-fast limit cycles in predator-prey models, Ecol. Model. 61 (1992), $287-308$.

[17] M. L. Rosenzweig and R. H. MacArthur, Graphical representation and stability conditions of predator-prey interactions, Am. Nat. 97 (1963), 209-223.

[18] Y. Shimazu et al., Some problems in ecology oriented enviromentology, J. Earth Sci. Nagoya Univ. 20 (1972), 31-89.

[19] P. Szmolyan, Transversal heteroclinic and homoclinic orbits in singular perturbation problems, J. Differential Equations 92 (1991), no. 2, 252-281, DOI 10.1016/0022-0396(91)90049-F. MR.1120905 (92e:58185)

[20] M. A. Teixeira, Perturbation Theory for Non-smooth Dynamical Systems, Encyclopedia of Complexity and Systems Science, Ed. G. Gaeta, Springer-Verlag, 2008. 